\title{
Brain Abscess after Glioblastoma Resection: An Unusual Case Report
}

\author{
Uday Raswan,, Nayil Khursheed, Rumana Makhdoomi, and Altaf Ramzan ${ }^{1}$
}

\begin{abstract}
Background: Glioblastoma (GBM) is the most common primary brain tumor. It carries a poor prognosis with a median survival of approximately 14 mo even with aggressive multi-modality therapy. After neurosurgical procedures, infection most commonly presents as meningitis, subdural empyema, or cerebral abscess. Once diagnosed, post-operative meningitis can often be treated with a course of intravenous antibiotics. In contrast, cases that involve a bone flap infection, subdural empyema, or cerebral abscess usually require a repeat operation.

Case Presentation: We present the case of a glioblastoma of the frontal lobe that was complicated by abscess formation at the primary operative site. Emergency surgical intervention was performed. The organism isolated from the brain abscess was Staphylococcus aureus.

Conclusion: Clinical suspicion of abscess should be kept in mind when patients present with sudden deterioration post-glioma resection. Combined surgical and medical therapy can manage such cases successfully.
\end{abstract}

Keywords: brain abscess; brain tumor; glioblastoma

G LIOBLASTOMA (GBM) is the most common primary brain tumor. It carries a poor prognosis with a median survival of approximately 14 mo even with aggressive multi-modality therapy [1]. After neurosurgical procedures, infection presents most commonly as meningitis, subdural empyema, or cerebral abscess. Once diagnosed, post-operative meningitis can often be treated with a course of intravenous antibiotics. In contrast, cases that involve a bone flap infection, subdural empyema, or cerebral abscess usually require a repeat operation [2]. We present a case of glioblastoma of the frontal lobe complicated by abscess formation at the primary operative site.

\section{Case Presentation}

An 80-year-old male operated on for left frontal glioblastoma multiforme (Fig. 1), two months prior months who was receiving radiotherapy and chemotherapy reported to the emergency department with fever and swelling on the previous craniotomy site of five days' duration. He also had persistent vomiting and headache for two days and altered sensorium for $12 \mathrm{~h}$. On neurologic examination his Glasgow Coma Scale (GCS) was 11. His pupils were normal in size and reactive to light. The skin over the craniotomy site was inflamed. In view of the kidney function tests a plain head computed tomography (CT) scan (Fig. 2) was done, which showed a hypodense mass at the previous tumor site (left frontal). There was peri-lesion edema and compression of frontal horn of ipsilateral ventricle. A diagnosis of brain abscess was made and surgical intervention was performed. After reflecting the skin incision subgaleal pus was visible. The bone flap was removed and pus collection was also observed underneath, extending through the subdural space and brain parenchyma. The abscess was excised and a meticulous debridement with saline, gentamicin, and hydrogen peroxide was done. The bone flap was not replaced. Cultures from the abscess revealed Staphylococcus aureus sensitive to vancomycin and ceftazidime; antibiotics were administered. Post-operative brain CT (Fig. 3) revealed total resolution of the abscess, however, brain edema was present. The patient recovered fully to GCS 15 . Histopathologic examination of the excised tissue confirmed it as abscess wall.

\section{Discussion}

In the past, infection was the major contributor to morbidity and mortality rates, occurring after almost all operations [1]. A $0.8 \%-7 \%$ incidence of post-operative infection

\footnotetext{
${ }^{1}$ Department of Neurosurgery, ${ }^{2}$ Department of Pathology, Sher-i-Kashmir Institute of Medical Sciences, Srinagar, Kashmir, India.
}

(C) Uday Raswan et al. 2017; Published by Mary Ann Liebert, Inc. This Open Access article is distributed under the terms of the Creative Commons License (http://creativecommons.org/licenses/by/4.0), which permits unrestricted use, distribution, and reproduction in any medium, provided the original work is properly credited. 


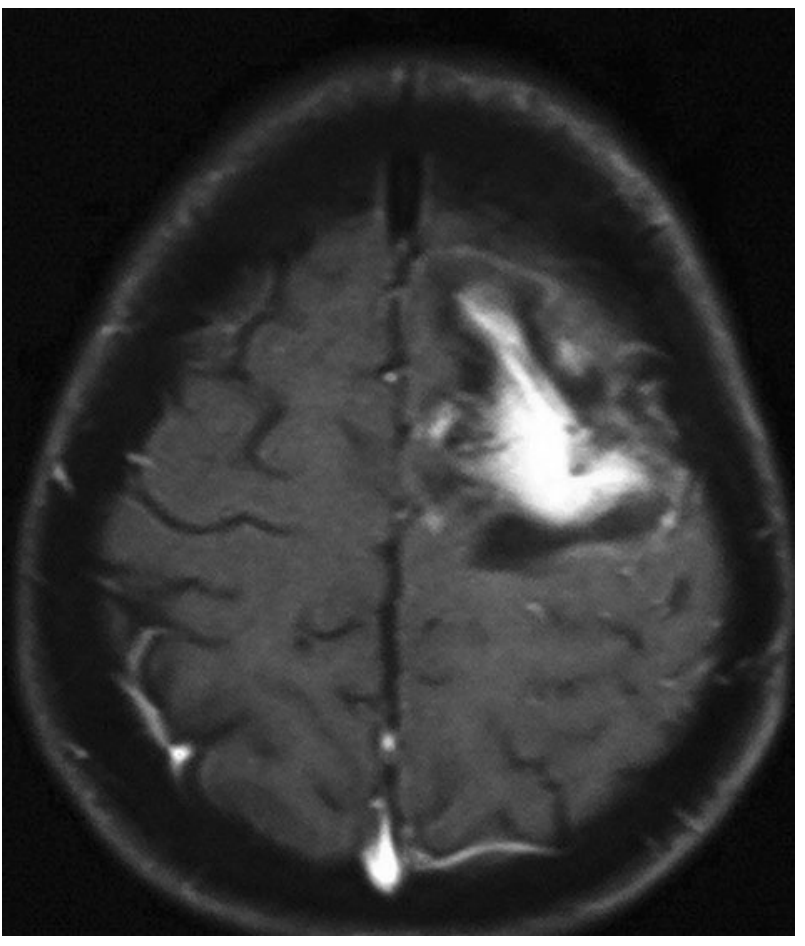

FIG. 1. Contrast magnetic resonance imaging (MRI) brain axial section shows left frontal irregularly enhancing lesion.

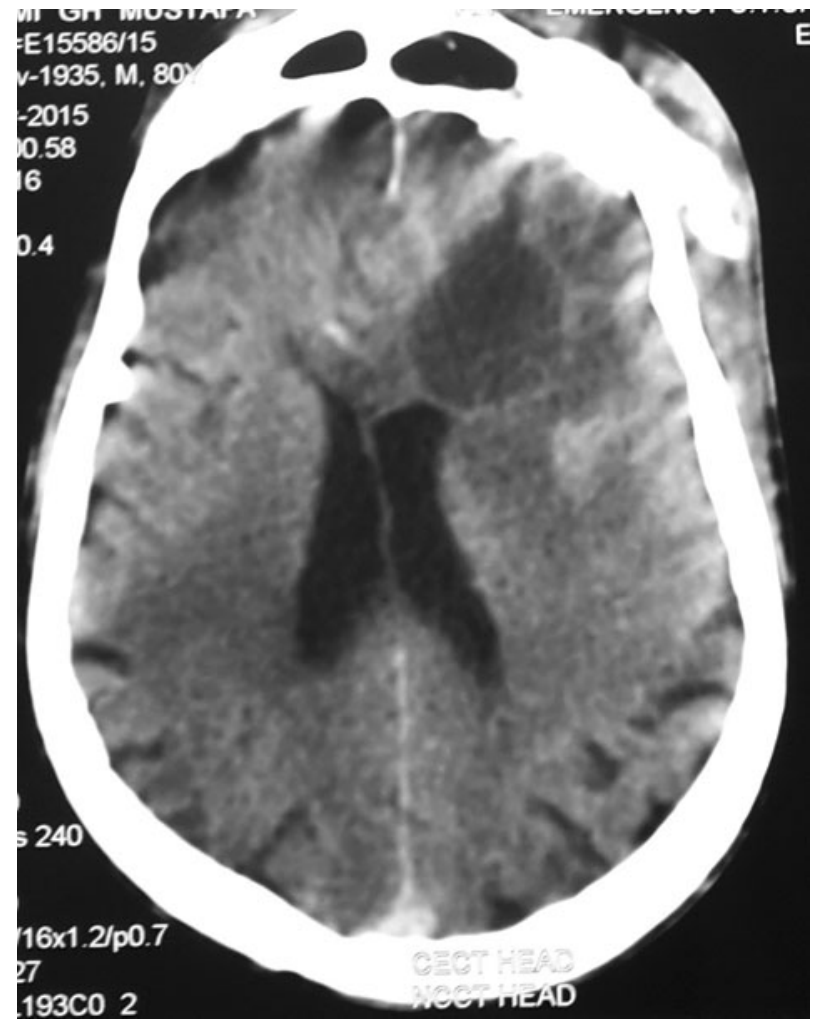

FIG. 2. Plain computed tomography (CT) of the shows well-defined globular hypodense lesion in left frontal lobe abutting the ventricle.

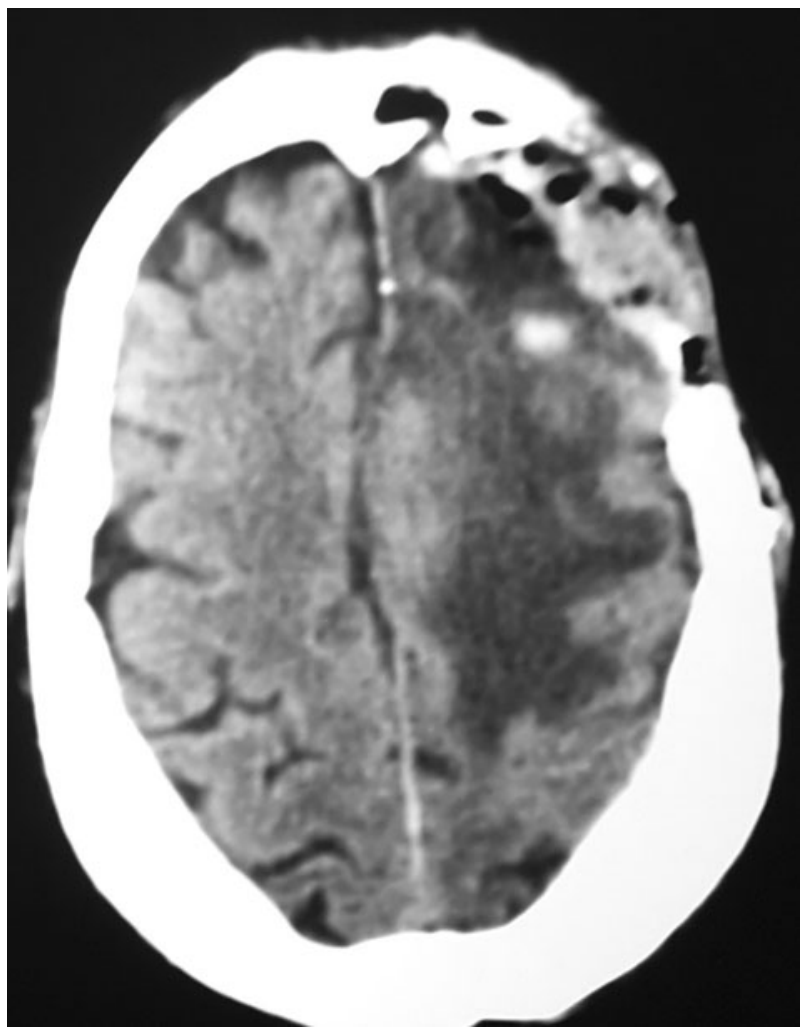

FIG. 3. Plain computed tomography (CT) of the head shows evidence of excision of the lesion but with features of brain edema.

has been reported in large series of neurosurgical procedures in which patients received pre-operative antibiotics prophylactically [2]. After neurosurgical procedures, infection most commonly presents as meningitis, empyema, wound/bone infection, or cerebral abscess [1].

In development of brain abscess, inoculation of an organism is required into the brain parenchyma in area of devitalized brain tissue or in a region with poor microcirculation. Histologically, there are four stages in brain abscess formation: early cerebritis (day 1-3), late cerebritis (day 4-9), early encapsulation (day 10-13), and late capsule stage (day 14 onward). Approximately two weeks are required for encapsulation, which is usually less complete on the medial or ventricular side because of poor vascular supply. In the pre-antibiotic era, the most common organism isolated from a brain abscess was Staphylococcus aureus, as in our patient. Now, streptococcus has replaced staphylococcus as the most common organism [3].

Clinically, brain abscesses present with features of rapidly expanding intra-cranial mass lesion that is increased intracranial pressure (ICP) in the form of constant progressive headache refractory to therapy, vomiting, papilledema, focal deficits, convulsions, meningismus, and altered sensorium. The classic triad of headache, focal neurologic deficits, and fever is found in only $25 \%$ of cases. The duration of symptoms is usually less than two weeks, with rapid onset and progression. Immunocompromised patients may have an insidious onset [4]. Our patient presented with rapid onset increased ICP.

The majority of post-surgical infections are caused by contamination of the incision with bacteria from the patient's 
skin. Host defense mechanisms represent the primary barrier to establishment of infection and these defenses may be impaired in patients undergoing craniotomy. Low concentrations of antibodies and complement contribute to make the brain less efficient than other organs at eradicating infections. Patients with malignant gliomas express a variety of immune defects, including increased secretion of immunosuppressive cytokines and an increased fraction of regulatory T-cells [5]. Additionally, many of the adjunctive therapies used for treating brain tumors, such as corticosteroids, chemotherapy, and radiation may result in immune compromise [3], and this seems to be possible explanation for development of abscess in our case. Also, malignant gliomas such as glioblastoma appear to induce systemic depression of the cellular immunity in patients that is notably severe [6]. Advanced age, hypoalbuminemia, diabetes mellitus, and poor functional status are the general risk factors.

Factors specific to craniotomy have been identified as increasing the risk of postoperative infection. Korinek [7] identified post-operative cerebrospinal fluid (CSF) leakage and early subsequent re-operation as independent risk factors, thus suggesting that careful attention to closure techniques and meticulous hemostasis potentially may result in lower rates of post-operative infections. Additional independent risk factors identified were: surgery lasting longer than four hours, emergency surgery, clean-contaminated and contaminated surgery, and neurosurgical intervention in the preceding month [2].

The management options for intra-cranial abscess are either a combination of surgery and prolonged antibiotic therapy or medical therapy alone. Walled off abscess larger than $3 \mathrm{~cm}$ diameter and a smaller deep-seated white matter abscess are unlikely to respond to medical treatment alone. Standard therapy for such lesions should be surgical evacuation followed by appropriate antibiotic therapy. Many authors recommended craniotomy and excision for abscesses that enlarge after two weeks of antibiotic therapy or that fail to shrink after three to four weeks of antibiotic therapy. Craniotomy is also recommended for multi-loculated abscesses and larger lesions with significant mass effect that are superficial and located in non-eloquent regions of the brain [3].

The non-surgical management of central nervous system (CNS) infections is restricted to patients who are neurologically intact, unable to undergo surgical procedure, and where the organism can be identified from other cultures. In these patients, brain abscess less than $2.5 \mathrm{~cm}$ in diameter without mid-line shift and a good response to antibiotic therapy may be treated successfully with prolonged intravenous antibiotic therapy [8].

\section{Conclusion}

Clinical suspicion of abscess should be kept in mind when patients present with fever and raised ICP after glioma resection. Combined surgical and medical therapy can manage such cases successfully.

\section{Author Disclosure Statement}

No competing financial interests exist.

\section{References}

1. De Bonis P, Albanese A, Lofrese G, et al. Postoperative infection may influence survival in patients with glioblastoma: Simply a myth? Neurosurgery 2011;69:864-869.

2. Dashti SR, Baharvahdat H, Spetzler RF, et al. Operative intracranial infection following craniotomy. Neurosurg Focus. 2008;24:E10.

3. Chowdhury FH, Haque MR, Sarkar MH, et al. Brain abscess: Surgical experiences of 162 Cases. Neuroimmunol Neuroinflamm 2015;2:153-161.

4. Sharma BS, Gupta SK, Khosla VK. Current concepts in the management of pyogenic brain abscess. Neurology India 2000;48:105-111.

5. Farrell Christopher J, Pisculli Mary L, Barker Frederick G. Post-operative infections of the head and brain. In: Youmans JR (ed): Neurological Surgery, 6th ed. Philadelphia, PA: WB Saunders, 2011: 560-569.

6. Dimovi I, Tasic D, Stefanovic I, Dimov D. New insights into molecular basis of glioblastoma multiforme and associated immunosuppression. Scientific Journal of the Faculty of Medicine in Niš 2013;30:165-184.

7. Korinek AM. Risk factors for neurological site infections after craniotomy: A prospective multicenter study of 2944 patients. The French Study Group of Neurosurgical Infections, the SHEP, and the C-CLIN Paris-Nord. Service Epidémiologie Hygiène et Prévention. Neurosurg. 1997;41:1073-1079.

8. Galbarriatu L, Rivero-Garvia M, Miranda D, et al. Postcraniotomy brain abscess and empyema by Clostridum perfringens: An unusual neurosurgical complication. Int $\mathrm{J}$ Case Rep Medicine 2013.Article ID 245718.

9. Duntze J, Litre CF, Bajolet O, et al. Clostridial brain abscess after glioblastoma resection: Case report and critical review of the literature. J Neurochirurgie 2009;55:569-572.

Addess correspondence to:

Dr. Nayil Khursheed

Department of Neurosurgery

SKIMS

Kashmir

India

E-mail: nayilkhursh@gmail.com

$\begin{aligned} & \text { Abbreviations Used } \\ \mathrm{CNS} & =\text { central nervous system } \\ \mathrm{CSF} & =\text { cerebrospinal fluid } \\ \mathrm{CT} & =\text { computed tomography } \\ \mathrm{GBM} & =\text { glioblastoma } \\ \mathrm{GCS} & =\text { Glasgow Coma Scale } \\ \mathrm{ICP} & =\text { intra-cranial pressure } \\ \mathrm{MRI} & =\text { magnetic resonance imaging }\end{aligned}$

Cite this article as: Raswan U, Khursheed N,

Makhdoomi R, and Ramzan A (2017) Brain abscess after glioblastoma resection: an unusual case report. Surgical Infections Case Reports 2:1, 69-71, DOI: 10.1089/ crsi.2016.0015 